\title{
Correction to: Infectious mononucleosis, immune genotypes, and non- Hodgkin lymphoma (NHL): an InterLymph Consortium study
}

\author{
Niquelle Brown Wadé ${ }^{1,2} \cdot$ Cindy M. Chang ${ }^{3}$. David Conti ${ }^{1,4}$. Joshua Millstein ${ }^{1,4}$. Christine Skibola ${ }^{5}$. \\ Alexandra Nieters ${ }^{6}$. Sophia S. Wang ${ }^{7}$. Silvia De Sanjose ${ }^{8,9}$. Eleanor Kane ${ }^{10}$. John J. Spinelli ${ }^{11,12}$. Paige Bracci ${ }^{13}$. \\ Yawei Zhang ${ }^{14}$. Susan Slager ${ }^{15}$. Jun Wang ${ }^{1,4}$. Henrik Hjalgrim ${ }^{16,17}$. Karin Ekstrom Smedby ${ }^{18}$. Elizabeth E. Brown ${ }^{19}$. \\ Ruth F. Jarrett ${ }^{20}$. Wendy Cozen ${ }^{1,4,21}$ (1) for the InterLymph Consortium Immunology and Infection Working Group
}

Published online: 29 March 2020

(c) Springer Nature Switzerland AG 2020

\section{Correction to: Cancer Causes \& Control https://doi.org/10.1007/s10552-020-01266-4}

Unfortunately, the word "Group" is missed in the author group of the original publication. It has been corrected by this erratum.

The original article can be found online at https://doi.org/10.1007/ s10552-020-01266-4.

\section{Wendy Cozen}

wcozen@med.usc.edu

1 Department of Preventive Medicine, Center for Genetic Epidemiology, Keck School of Medicine, University of Southern California, Los Angeles, CA, USA

2 Cigna Health and Life Insurance Company (Cigna), Bloomfield, CT, USA

3 Division of Population Health Sciences, Center for Tobacco Products, Food and Drug Administration, Bethesda, MD, USA

4 USC Norris Comprehensive Cancer Center, Keck School of Medicine, University of Southern California, Los Angeles, CA, USA

5 Department of Hematology and Medical Oncology, Emory University School of Medicine, Atlanta, GA, USA

6 Center for Chronic Immunodeficiency (CCI), University Medical Center Freiburg, University of Freiburg, Freiburg, Germany

7 Department of Computational and Quantitative Medicine, City of Hope Comprehensive Cancer Center, Duarte, CA, USA

8 Sexual and Reproductive Health, PATH, Seattle, WA, USA

9 Centro de Investigación Biomédica en Red: Epidemiología y Salud Pública (CIBERESP), Madrid, Spain

10 Department of Health Sciences, University of York, York YO10 5DD, UK
Publisher's Note Springer Nature remains neutral with regard to jurisdictional claims in published maps and institutional affiliations.
11 Population Oncology, BC Cancer Agency, Vancouver, Canada

12 School of Population and Public Health, University of British Columbia, Vancouver, BC, Canada

13 Department of Epidemiology and Biostatistics, University of California at San Francisco, San Francisco, CA, USA

14 Department of Surgery, Yale School of Medicine and Yale School of Public Health, New Haven, CT, USA

15 Department of Epidemiology, Mayo Clinic, Rochester, MN, USA

16 Department of Epidemiology Research, Statens Serum Institut, Copenhagen, Denmark

17 Department of Haematology, Rigshospitalet, Copenhagen, Denmark

18 Karolinska Institutet, Sweden University Hospital, Karolinska University, Stockholm, Sweden

19 Department of Pathology, O’Neal Comprehensive Cancer Center, University of Alabama at Birmingham, Birmingham, AL, USA

20 MRC-University of Glasgow Centre for Virus Research, Glasgow, Scotland

21 Department of Pathology, Keck School of Medicine, University of Southern California, Los Angeles, CA 90033, USA 